\title{
Autoestima em estudantes adolescentes de duas escolas estaduais da Cidade de
}

\section{Nazaré da Mata - PE}

\author{
Self-esteem in adolescent students from two state schools in the City of Nazaré da Mata - PE \\ Autoestima en estudiantes adolescentes de dos escuelas públicas de la Ciudad de Nazaré da Mata - \\ PE
}

\begin{abstract}
Resumo
A autoestima está associada à qualidade de vida, pois ela é tida como um importante indicador de saúde mental, e é conceituada como um conjunto de sentimentos e pensamentos do indivíduo sobre o seu próprio valor, competência e adequação, que repercute em atitude positiva ou negativa em relação a si. Logo, torna - se interessante à avaliação dela por ser a amostra composta de adolescentes, pois a adolescência é um período de grandes alterações físicas, psicológicas e sociais. O objetivo do trabalho em questão é avaliar a autoestima de estudantes adolescentes. A amostra foi composta por 36 estudantes de duas escolas públicas do município de Nazaré da Mata. Utilizamos como método à aplicação da Escala de Avaliação da Autoestima de Rosenberg (1979) adaptada para o português por Hutz (2011) e uma ficha para coleta de dados a fim de caracterizar os grupos investigados. Para análise dos dados usamos o programa SPSS 20.0, e para a análise descritiva, foi observada a média e desvio padrão dos grupos. Para analisar se haviam diferenças estatisticamente significativas entre os grupos, utilizamos o teste t para amostras independentes. $\mathrm{O}$ resultado mostrou que não houve diferenças estatisticamente significativas entre os grupos, apesar da média da escola de tempo integral ser maior que o grupo da escola de tempo parcial.
\end{abstract}

Palavras-chave: Autoestima; Estudantes; Adolescentes.

\begin{abstract}
Self-esteem is associated with quality of life, as it is seen as an important mental health indicator, and it is conceptualized as a set of feelings and thoughts of the individual about his own value, competence and adequacy, which has a positive or negative attitude. negative towards you. Therefore, it is interesting to evaluate it because it is a sample of adolescents, as adolescence is a period of great physical, psychological and social changes. The objective of the work in question is to assess the self-esteem of adolescent students. The sample consisted of 36 students from two public schools in the municipality of Nazaré da Mata. We used as a method to apply the Rosenberg's Self-Esteem Assessment Scale (1979) adapted to Portuguese by Hutz (2011) and a data collection form in order to characterize the investigated groups. For data analysis, we used the SPSS 20.0 program, and for descriptive analysis, the mean and standard deviation of the groups were observed. To analyze whether there were statistically significant differences between the groups, we used the $t$ test for independent samples. The result showed that there were no statistically significant differences between the groups, despite the average of the full-time school being higher than the group of the part-time school.
\end{abstract}

Keywords: Self-esteem; Students; Teenagers. 


\section{Resumen}

La autoestima se asocia con la calidad de vida, ya que se ve como un indicador importante de salud mental, y se conceptualiza como un conjunto de sentimientos y pensamientos del individuo sobre su propio valor, competencia y adecuación, que tiene un impacto positivo o negativo. actitud negativa hacia ti. Por tanto, es interesante evaluarlo porque se trata de una muestra de adolescentes, ya que la adolescencia es un período de grandes cambios físicos, psicológicos y sociales. El objetivo del trabajo en cuestión es evaluar la autoestima de los estudiantes adolescentes. La muestra estuvo compuesta por 36 estudiantes de dos escuelas públicas del municipio de Nazaré da Mata. Utilizamos como método para aplicar la Escala de Autoestima de Rosenberg (1979) adaptada al portugués por Hutz (2011) y un formulario de recolección de datos para caracterizar los grupos investigados. Para el análisis de los datos se utilizó el programa SPSS 20.0, y para el análisis descriptivo se observó la media y la desviación estándar de los grupos. Para analizar si existían diferencias estadísticamente significativas entre los grupos, utilizamos la prueba t para muestras independientes. El resultado mostró que no hubo diferencias estadísticamente significativas entre los grupos, a pesar de que el promedio de la escuela a tiempo completo fue más alto que el del grupo de la escuela a tiempo parcial.

Palabras clave: Autoestima; Estudiantes; Adolescentes.

\section{Introdução}

A autoestima é entendida como um conjunto de pensamentos e sentimentos do sujeito no que se refere ao seu próprio valor, adequação e competência, que se ajuíza em uma atitude negativa ou positiva em relação a ele mesmo (Rosenberg, 1965).

Definir a autoestima é uma tarefa imensamente complicada, pois abrange compreensão do "mundo interno", do mundo externo e atribuições de valor a crenças. Aborda - se de um constructo particular e intrínseco que sofre forte influência do contexto cultural e social em que está inserido o sujeito (Schultheisz, 2015).

A autoestima é analisada como um dos essenciais preditores de efeitos benéficos na vida adulta e na adolescência, tendo ligação em campos como relacionamentos interpessoais, desempenho acadêmico e sucesso ocupacional (Trzesniewski, Donnellan \& Robins, 2013). É indicada como um relevante apontador de saúde mental por gerar interferência nas condições psicológicas, sociais e afetivas dos indivíduos (Altintas, Gallouj \& Guerrien, 2013). Deste modo, pode intervir na qualidade de vida, saúde e bem estar da população geral (Schultheisz, 2015).

A adolescência compõe a faixa etária em que o sujeito exibe transformações emocionais, cognitivas e físicas que, por sua vez, intervém na constituição de sua autoestima (Orth, 2010). A análise da autoestima na adolescência é relevante, pois nota-se que nessa faixa etária, além dessas transformações que geram influência na composição da autoestima, há uma da afinidade positiva da autoestima com a saúde mental, existem relações elevadas e significativas entre elas, aprovação social e rendimento escolar (Steinberg, 1999).

A escola é um espaço estimado para reflexões a respeito das questões que abrangem crianças e adolescentes, educadores, pais, filhos, assim como os vínculos que se dão na sociedade. É ainda nesse universo onde a promoção da cidadania, a socialização, a formação de opiniões, atitudes e desenvolvimento pessoal podem ser prejudicados ou desenvolvidos (Marriel, 2006). No ambiente escolar os alunos devem estar motivados (Melo, Souza, Silva, 2020), pois, diversos fatores, como o sono, podem interferir na aprendizagem (Oliveira et al., 2019).

É de suma importância à avaliação da autoestima dos alunos das escolas de ensino de tempo integral e tempo parcial, pois são ambientes diferentes. Ao comparar ambas as instituições, percebe-se que os alunos da primeira escola passam mais tempo dentro do âmbito escolar, por conta das cargas horárias extensas e isto pode estar associado há ter mais tempo para aprender mais coisas e maior aprofundamento. Vale ressaltar a maior interação entre os discentes e outros componentes da escola, e menor interação com os pais, que contribuem para influenciar na educação dos seus filhos (Gonçalves, 2006).

As escolas que funcionam em tempo integral apresentam melhores estruturas, docentes mais capacitados e que são mais bem remunerados, com melhor nível educacional (Brandão, 2009), pois além das aulas tradicionais em sala de aula, muitas delas oferecem para completar a educação, diversas atividades extracurriculares (Cavaliere, 2009). Outro ponto 
importante é que muitas apresentam uma quantidade maior de aulas de educação física comparada à escola de ensino parcial (CENP/SP, 2011) e, estes fatores podem gerar influência ao analisar a autoestima.

Diante disso, o estudo em questão tem por finalidade analisar e comparar a autoestima dos estudantes de duas instituições públicas do munícipio de Nazaré da Mata - PE, sendo uma de tempo integral e outra de tempo parcial.

\section{Metodologia}

O estudo em questão trata-se de um estudo descrito, quantitativo e de corte transversal. A população do estudo é de 36 estudantes matriculados no $1^{\circ}$ ano do ensino médio de duas escolas públicas.

O estudo foi aprovado pelo Comitê de Ética em Pesquisa com Seres Humanos da Universidade Federal de Pernambuco (UFPE) sob o parecer n. 1.782.873.

Para participarem da pesquisa os sujeitos deveriam estar matriculados em ambas as escolas no $1^{\circ}$ ano do ensino médio, ter entre 14 e 17 anos, ter entregado o Termo de Consentimento Livre e Esclarecido (TCLE) e o Termo de Assentimento Livre e Esclarecido (TALE) assinados ao pesquisador. Foram excluídos da pesquisa, os adolescentes com idade inferior a 14 anos e/ou superior a 17 anos; que não tiverem entregado o TCLE e o TALE assinados e aqueles com incapacidade mental e outros que se julgue impossibilitados de responder ao questionário.

A autoestima foi avaliada com a utilização da escala de avaliação da autoestima de Rosenberg (1979). Ela apresenta dez afirmações, referentes à autoaceitação e a um conjunto de sentimentos de autoestima que avalia a autoestima global. A escala é do tipo Likert e apresenta quatro pontos que varia em concordo totalmente, concordo, discordo e discordo totalmente, onde os itens podem ser respondidos. Neste estudo foi utilizada a versão adaptada para o português por Hutz (2011) que manteve a avaliação como uma medida unidimensional com características psicométricas equivalentes às do instrumento original.

Para caracterização da amostra estudada, os adolescentes responderam a um questionário adaptado a partir do trabalho de Balhau (2011).

Os dados foram tabulados no programa Microsoft Office Excel 2010. Para análise dos dados foi utilizado o programa SPSS 20.0. Para a análise descritiva, foi observada a média e desvio padrão e utilizamos o Teste $\mathrm{T}$ para amostras independentes, a fim de avaliar se existiam diferenças estatisticamente significativas entre os dois grupos investigados.

\section{Resultados}

Quando se refere à média idade da amostra do presente estudo, tem-se que a escola de tempo integral apresentou um menor media $(M=15,50 ; \mathrm{DP}=0,51)$ se comparada com a escola de tempo parcial $(\mathrm{M}=15,60 ; \mathrm{DP}=0,50)$. Para a dimensão gênero em ambas as escolas 55,50\% representou a média para o gênero masculino e 44,50\% para o gênero feminino, como representado na tabela 1 . 
Tabela 1 - Caracterização dos Participantes.

\begin{tabular}{lccccc}
\hline & & \multicolumn{2}{c}{ ETP } & \multicolumn{2}{c}{ ETI } \\
& & Média & $\%$ & Média & $\%$ \\
\hline Idade & & $15,60(0,50)$ & & $15,50(0,51)$ & \\
& & & & & \\
& Feminino & 8,00 & $44,50 \%$ & 8,00 & $44,50 \%$ \\
& Masculino & 10,00 & $55,50 \%$ & 10,00 & $55,50 \%$ \\
\hline
\end{tabular}

Fonte: Autores (2021).

Os resultados obtidos ao nível da autoestima indicam que os participantes do grupo de alunos da escola de tempo parcial apresentaram um valor médio de autoestima mais elevado $(\mathrm{M}=29,83$; $\mathrm{DP}=3,86)$, comparado ao grupo de alunos da escola de tempo integral $(\mathrm{M}=31,61$; $\mathrm{DP}=2,40)$. As diferenças entre os valores médios de autoestima de ambos os grupos apresentados não são estatisticamente significativas $(\mathrm{p}=0,109)$, como é representado na tabela 2.

Tabela 2 - Comparações de médias de autoestima entre os grupos.

\begin{tabular}{cccc}
\hline \multirow{2}{*}{ Variáveis } & \multicolumn{2}{c}{ Autoestima } & Teste $t$ \\
\cline { 2 - 4 } & Média & Desvio Padrão & $P$ \\
\hline Escola Tempo Parcial & 29,83 & 3,86 & 0,109 \\
Escola Tempo Integral & 31,61 & 2,40 & \\
\hline
\end{tabular}

Fonte: Autores (2021).

Segue - se ainda, os resultados expressos em percentuais obtidos através do questionário adaptado de Balhau (2011). Na tabela 3 a RCT representa a relação com os colegas de turma, a RAE a relação com os amigos da escola, a FSAEF, que é a frequência semanal de aulas de educação Física, a AFFAE que é a realização de atividade física fora do ambiente escolar e, por fim a RPEF, que representa a relação com o professor de educação Física. 
Tabela 3 - Percentual dos dados coletados no questionário.

\begin{tabular}{|c|c|c|}
\hline \multirow{2}{*}{ Variáveis } & $\overline{\operatorname{ETP}}(\mathrm{n}=18)$ & $\operatorname{ETI}(n=18)$ \\
\hline & $\%$ & $\%$ \\
\hline \multicolumn{3}{|l|}{ RCT } \\
\hline Nem boa, nem má & $5,50 \%$ & - \\
\hline Boa & $66,70 \%$ & $55,60 \%$ \\
\hline Muito boa & $27,80 \%$ & $44,40 \%$ \\
\hline \multicolumn{3}{|l|}{ RAE } \\
\hline Nem boa, nem má & $38,90 \%$ & $5,50 \%$ \\
\hline Boa & $22,20 \%$ & $55,60 \%$ \\
\hline Muito boa & $38,90 \%$ & $38,90 \%$ \\
\hline \multicolumn{3}{|l|}{ FSAEF } \\
\hline 1 aula & $100 \%$ & - \\
\hline 2 aulas & - & $100 \%$ \\
\hline \multicolumn{3}{|l|}{ AFFAE } \\
\hline Não & $38,90 \%$ & $27,80 \%$ \\
\hline Sim & $61,10 \%$ & $72,20 \%$ \\
\hline \multicolumn{3}{|l|}{ RPEF } \\
\hline Boa & $50,00 \%$ & $66,70 \%$ \\
\hline Muito boa & $38,90 \%$ & $33,30 \%$ \\
\hline Ruim & $11,10 \%$ & - \\
\hline
\end{tabular}

$\%=$ Resultado expresso em percentual

Fonte: Autores (2021).

\section{Discussão}

O objetivo deste artigo foi avaliar e comparar a autoestima dos adolescentes estudantes das escolas de ensino em tempo integral e parcial. Ele evidenciou que não houve diferença estatisticamente significativa entre os grupos, como pode ser observado na tabela $2(\mathrm{p}=0,109)$, mas, baseado na média, a escola integral apresentou uma maior pontuação $(31,61)$, sugerindo uma melhor autoestima, quando comparada a escola de tempo parcial $(29,83)$. A discussão desta pesquisa será dirigida baseada na diferença entre as médias dos dois grupos investigados.

A autoestima está relacionada ao quão intensamente o individuo está insatisfeito ou satisfeito em relação às circunstâncias vividas (Hutz, Zanom, 2011). A literatura afirma que há uma relação entre autoestima e aprovação social (STEINBERG, 1999), relações interpessoais (Bahls, 2002), habilidades sociais (Del Prette \& Del Prette, 2008).

O mesmo autor (Bahls, 2002) afirma que na puberdade constata-se a existência de dificuldades relacionadas às relações interpessoais, dificuldade de interação com outros adolescentes, provavelmente pelo sentimento de baixa autoestima. Nesse sentido, 44,40\% dos alunos da instituição de tempo integral afirmaram ter uma relação "muito boa relação" com os colegas de turma, enquanto $27,80 \%$ dos alunos da escola de ensino parcial assinalaram a mesma proposição (tabela 3 ).

Quando se refere à dimensão relação amigos da escola (tabela 3), observa-se que 55,60\% dos alunos da escola de tempo integral afirmaram ter "boa relação" com eles, já na instituição de tempo parcial o percentual de alunos para essa mesma classificação foram $22,20 \%$. Este dado mostra que talvez o grupo da escola de período integral se relacionava melhor com os 
colegas de turma e amigos da escola e tinham maiores habilidades e aprovação social, podendo estar relacionado à maior autoestima dos alunos desta instituição.

Hewitt (2009) alega que a autoestima aparece do reconhecimento positivo por pares e outros analisados significativos como docentes e pais. Hutz, Zanon e Vazquez (2014) também sugerem que a alta autoestima nasce pela influência do ambiente e pelo do reconhecimento positivo de pais e professores. O percentual de alunos que apresentaram boa relação com o professor de educação física foi $66,70 \%$ na escola de tempo parcial, enquanto a mesma proposição teve o percentual de $50,00 \%$ para o grupo da escola de tempo parcial (tabela 3). Supomos que por apresentarem melhores percentuais na dimensão relação com o professor de educação física, os alunos da escola de ensino integral apresentaram melhor autoestima.

Em um de seus estudos, Sonstroem (1994) destacou que indivíduos que praticam exercício físico apresentam níveis mais positivos de autoestima global quando comparados com indivíduos que não pratica. A práxis da atividade física e do exercício decorre em evidentes benefícios psicológicos, designadamente, na melhora do funcionamento cognitivo, autoestima e imagem corporal (Cruz et al., 1996).

Segundo o estudo concretizado por Birkeland e colaboradores (2012) com a colaboração de aproximadamente 1.000 adolescentes noruegueses, o aumento da autoestima durante o período da adolescência estava associado a variáveis como a prática de atividades físicas e uma boa relação com os pais, além do maior percentual de praticantes de atividade física fora do ambiente escolar, que segundo a literatura melhora a autoestima (Ortega, 2008).

Feliciano (2012) em um dos seus estudos com uma amostra de adolescentes de 12 a 17 anos, mostrou que os adolescentes que apresentam uma autoestima mais elevada eram os que praticavam algum tipo de atividade física. Como pode ser observado na tabela 3, o percentual dos alunos da escola de tempo integral que afirmou praticar atividade física fora do ambiente escolar foi de 72,20\%, e 61,10\% corresponde ao percentual de alunos da escola de tempo parcial para a mesma afirmativa. Isto mostra que os alunos da instituição de ensino integral são mais ativos e, como consequência, talvez apresentassem níveis maiores de autoestima.

Vale destacar também que na escola de tempo integral, os alunos tinham duas aulas semanais, comparando com a escola de tempo parcial, que apresentavam apenas uma (tabela 3). Com um número maior de aulas, talvez os alunos da escola de tempo integral tivessem o maior número de aulas práticas, e quem sabe este fator, pudesse colaborar para incremento da autoestima deles.

\section{Considerações Finais}

O estudo em questão procurou analisar a autoestima em relação a estudantes adolescentes de duas escolas públicas, sendo uma de tempo parcial e outra de tempo integral. Foi verificado que não existem diferenças significativamente estatísticas entre os dois grupos investigados.

O corrente estudo ainda reconhece a relevância de aprofundar a investigação que envolva o estudo da autoestima seguindo abordagens qualitativas, com uma amostra maior e observando outras variáveis, pois sabe - se que as modificações que acontecem nesta fase da vida podem interferir no comportamento dos indivíduos.

\section{Referências}

Altintas, E., Gallouj, K. \& Guerrien, A. (2012). Social support, depression and self-esteem in older persons: cluster analysis results. In Annales MedicoPsychologiques. 170(4), 256-62). 21 Street Camille Desmoulins, Issy, 92789 Moulineaux Cedex 9, France: Masson Editeur.

Balhau, J. R. C., Cunha, M. O., \& Marques, M. C. (2011). A Vinculação e a Auto-Estima em Jovens Não-Institucionalizados Vs. Institucionalizados (Master's thesis, ISMT).

Birkeland, M. S., Melkevik, O., Holsen, I., \& Wold, B. (2012). Trajectories of global self-esteem development during adolescence. Journal of adolescence, 35(1), 43-54. 
Brandão, Z. (2009). Escola de tempo integral e cidadania escolar. Em aberto, 21(80).

Cavaliere, A. M. (2009). Escolas de tempo integral versus alunos em tempo integral. Em aberto, 21(80).

Del Prette, Z. A. P., \& Del Prette, A. (2008). Um sistema de categorias de habilidades sociais educativas. Paidéia (Ribeirão Preto), 18(41), 517-530.

Gonçalves, A. S. (2006). Reflexões sobre educação integral e escola de tempo integral. Cadernos Cenpec| Nova série, 1(2).

Feliciano, I. P., \& Afonso, R. M. (2012). Estudo sobre a auto-estima em adolescentes dos 12 aos 17 anos. Psicologia, Saúde e Doenças, $13(2), 252-265$.

Hewitt, J. P. (2009). Self-esteem.

Hutz, C. S., \& Zanon, C. (2011). Revisão da apadtação, validação e normatização da escala de autoestima de Rosenberg. Avaliacao Psicológica: Interamerican Journal of Psychological Assessment, 10(1), 41-49.

Hutz, C. S. (2014). Avaliação em psicologia positiva. Artes Médicas Editora.

Marriel, L. C., Assis, S. G., Avanci, J. Q., \& Oliveira, R. V. (2006). Violência escolar e auto-estima de adolescentes. Cadernos de pesquisa, 36(127), 35-50. Melo, L. B. C., Souza, M. A., \& Silva, J. G. M. (2020). Motivação para a aprendizagem em crianças escolares: revisão narrativa. Revista Eletrônica Acervo Científico, 10, e3636.

Oliveira, W. A., Silva, L. K. T. M. D., Alves, S. J. D. O., Silva, J. G. M., \& Pinto, F. C. M. (2019). Sleep quality influence on learning in science teaching. Revista Psicopedagogia, 36(109), 73-86.

Ortega, F. B., Ruiz, J. R., Castillo, M. J., \& Sjöström, M. (2008). Physical fitness in childhood and adolescence: a powerful marker of health. International journal of obesity, 32(1), 1-11.

Orth, U., Trzesniewski, K. H., \& Robins, R. W. (2010). Self-esteem development from young adulthood to old age: a cohort-sequential longitudinal study. Journal of personality and social psychology, $98(4), 645$.

Rosenberg, M. (2015). Society and the adolescent self-image. Princeton university press.

Rosenberg, M., Schooler, C., \& Schoenbach, C. (1989). Self-esteem and adolescent problems: Modeling reciprocal effects. American sociological review, 1004-1018.

Schultheisz, T. S. D. V., \& Aprile, M. R. (2013). Autoestima, conceitos correlatos e avaliação. Revista Equilíbrio Corporal e Saúde, 5(1).

Sonstroem, R. J., Harlow, L. L., \& Josephs, L. (1994). Exercise and self-esteem: Validity of model expansion and exercise associations. Journal of Sport and Exercise psychology, 16(1), 29-42.

Trzesniewski, K. H., Donnellan, M. B., \& Robins, R. W. (2013). Development of self-esteem. Self-esteem, 60-79. 